Mustafa Rabeei, et al., Lpvioid - a LPV identification toolbox for matlab: recent and novel techniques, pp. 1637 - 1659

\title{
LPVIOID- A LPV IDENTIFICATION TOOLBOX FOR MATLAB: RECENT AND NOVEL TECHNIQUES
}

\author{
Mustafa Rabeei ${ }^{*}$, Hossam S. Abbas and Mohamed M. Hassan
}

Electrical Engineering Department, Assiut University, Assiut, Egypt.

Received 19 May 2013, accepted 25 June 2013

\begin{abstract}
In this paper a system identification toolbox for MATLAB is introduced, including a user friendly graphical user interface. The toolbox is appropriate for the identification of systems in discrete-time linear parameter varying (LPV) form. Using LPVIOID1 it is possible to identify input-output models in open-loop and closed-loop settings based on experimental data. It comprises several recent LPV identification techniques. Furthermore, a novel method for identifying unstable plants in closed-loop is proposed. The toolbox is equipped with several tools for model validation. Examples for illustration are included.
\end{abstract}

Keywords: Linear parameter varying systems, system identification, non-linear modelling.

\section{Introduction}

The field of system identification is pushed by the continuous need for accurate and efficient models for industrial applications. The identification methods to give models from input-output data have been applied successfully to linear time-invariant (LTI) systems. However, real systems are often nonlinear or have a time-varying nature. Therefore, approximating these systems by LTI models may result in a large error. On the other hand, identifying nonlinear models for these systems introduces complexities in terms of modeling and control synthesis.

The class of linear parameter-varying (LPV) systems can form an intermediate step between LTI and nonlinear/time-varying plants. In LPV representations, the signal relations are considered to be linear just as in the LTI case, but the parameters are assumed to be functions of an online measurable time-varying signal, the so-called scheduling variable. Therefore LPV models can describe a large class of nonlinear/time-varying systems in an attractive structure allowing based on linear control methods the use of LPV control-synthesis approaches, e.g. [3, 11], to control efficiently these systems. This has encouraged researches to develop techniques for LPV identification. Methods taking this approach can be either based on state-space models, see e.g. [15], or on input-output models [4]. From a practical point of view, the latter appear to be more promising [16]. Identification techniques based on input-output models have received recently considerable attention with many applied results $[4,5,8,1,7,13]$, as they are based on the extension of the well defined LTI Prediction Error Framework (PEM), [10], and enable model structure

\footnotetext{
${ }^{1}$ https://sites.google.com/site/mustafarabeei/home/lpvioid-toolbox

* Corresponding author.

E-mail address: eng.mustafa.rabeay@gmail.com
} 
Mustafa Rabeei, et al., Lpvioid - a LPV identification toolbox for matlab: recent and novel techniques, pp. 1637 - 1659

selection and the stochastic analysis of parameter estimation in a computationally attractive manner, [13].

The toolbox, LPVIOID, presented in this paper can be utilized to identify LPV inputoutput (LPV-IO) models in open-loop and closed-loop using the most recent identification techniques in the literature. These include the least squares (LS) method $[4,13]$, the basic instrumental variables (IV) method $[5,13]$ and the refined instrumental variables (RIV) as well as the simplified refined instrumental variables (SRIV) methods [8, 14]. Note that all these techniques assume stable processes. Therefore, the LPVIOID has been accomplished with a novel identification technique based on the LS, IV and an iterative LS methods for identifying unstable LPV-IO plants from measurements in closed-loop. The toolbox enables the user to identify LPV-IO models using different types of model structures including Auto-Regressive with exogenous input (ARX), Output-Error (OE) and BoxJenkins (BJ) types along with several ways for model validation. Additionally there is a user support in terms of graphical tools for both model identification and evaluation as well as support for bookkeeping of the whole identification session (including models and data). Finally it is worth to mention that, to the best of the authors knowledge, this is the first toolbox for LPV-IO identification with these attributes. Next the features offered by the LPVIOID toolbox are summarized:

1. It allows the user to select from different identification methodologies the suitable way to identify an LPV-IO model with different model structures (including ARX, $\mathrm{OE}$ and BJ types) given an informative measured input-output data set, process and noise models orders and basis (scheduling) functions.

2. It enables the user to identify stable/unstable plants in open-/closed-loop configurations.

3. It offers different identification methods including LS, IV, RIV, SRIV and iterative LS.

4. It enables the user to evaluate the identified model using several ways in time and frequency domains.

5. It provides a friendly graphical user interface using mouse-click operations.

6. It includes bookkeeping facilities, e.g. saving the the identification session, including data sets; models and other parameters, to a file such that it can be retrieved later, as well as user-guide documentation.

The paper is organized as follows: Section 2 reviews briefly the LPV-IO identification methods incorporated in the toolbox. Section 3 introduces a novel identification technique for identifying unstable LPV-IO models in closed-loop. The Main parts of the LPVIOID toolbox are described in Section 4 as well as the usage of all facilities and tools of the toolbox. Illustrative examples are presented in section 5. Finally, section 6 includes a summary and future work suggestions.

\section{LPV-IO identification}

In this section the identification methods incorporated in the LPVIOID toolbox are briefly described. The concepts of data generating LPV system, model representation and the definition of LPV-IO identification problem are reviewed.

Journal of Engineering Sciences, Assiut University, Faculty of Engineering, Vol. 41, No. 4, July, 2013,E-mail address: jes@aun.edu.eg 
Mustafa Rabeei, et al., Lpvioid - a LPV identification toolbox for matlab: recent and novel techniques, pp. 1637 - 1659

\section{1. Data generating LPV system}

For identification in open-loop, Fig. 1 shows a single-input single-output (SISO) discrete-time data-generating LPV system defined by

$$
A_{0}\left(p_{k}, q^{-1}\right) y(k)=B_{0}\left(p_{k}, q^{-1}\right) q^{-\tau} d(k)+A_{0}\left(p_{k}, q^{-1}\right) w(k)
$$

where $u(k), y(k)$ and $p(k) \in \mathrm{P}_{p}$ are system input, noisy output and scheduling signal, respectively, at a sampling instant $k, \mathrm{P}_{p}$ is a compact set representing the scheduling set of the LPV system, $w(k)$ is an additive noise with bounded spectral density, $q^{-1}$ is the backward time-shift operator such that $q^{-1} u(k)=u(k-1) q^{-1}$ and $\tau_{d}>0$ input delay. $A_{0}\left(p_{k}, q^{-1}\right)$ and $B_{0}\left(p_{k}, q^{-1}\right)$ are time-varying polynomials of degrees $n_{a}$ and $n_{b}\left(n_{b}+\tau_{d} \leq n_{a}\right)$, respectively, given by

$$
\begin{aligned}
& A_{0}\left(p_{k}, q^{-1}\right)=1+a_{1}^{0}\left(p_{k}\right) q^{-1}+\ldots+a_{n_{a}}^{0}\left(p_{k}\right) q^{-n_{a}}, \\
& B_{0}\left(p_{k}, q^{-1}\right)=b_{0}^{0}+b_{1}^{0}\left(p_{k}\right) q^{-1}+\ldots+b_{n_{b}}^{0}\left(p_{k}\right) q^{-n_{b}},
\end{aligned}
$$

where $a_{i}^{0}\left(p_{k}\right), i=1, \ldots, n_{a}$ and $b_{j}^{0}\left(p_{k}\right), j=0, \ldots, n_{b}$ are time-varying coefficients assumed to be non-singular on $\mathrm{P}_{p}$ with static dependence on $p(k)$, i.e. dependence on $p$ at sampling instant $k$ only. Note that $G_{0}$ in Fig. 1 indecates the process to be identified which is given by

$$
G_{0}\left(p_{k}, q^{-1}\right): A_{0}\left(p_{k}, q^{-1}\right) y_{0}(k)=B_{0}\left(p_{k}, q^{-1}\right) u(k)
$$

where $y_{0}(k)$ denotes noise-free output. Furthermore, $H_{0}$ in Fig. 1 indicates the noise process, which is represented by a discrete-time autoregressive moving average (ARMA), [10], model:

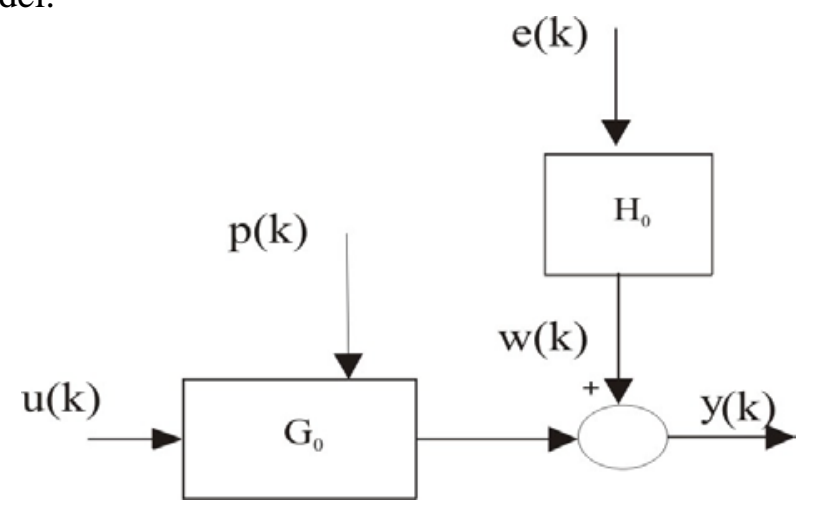

Fig. 1. open-loop system

Journal of Engineering Sciences, Assiut University, Faculty of Engineering, Vol. 41, No. 4, July, 2013,E-mail address: jes@aun.edu.eg 
Mustafa Rabeei, et al., Lpvioid - a LPV identification toolbox for matlab: recent and novel techniques, pp. 1637 - 1659

$$
w(k)=H_{0}\left(q^{-1}\right) e(k)=\frac{B_{H_{0}}\left(q^{-1}\right)}{A_{H_{0}}\left(q^{-1}\right)} e(k),
$$

where $e(k)$ is a zero-mean, discrete-time white noise process with a normal distribution $\mathrm{N}\left(0, \mu^{2}\right)$ where $\mu^{2}$ is the variance. $A_{H_{0}}\left(q^{-1}\right)$ and $B_{H_{0}}\left(q^{-1}\right)$ are monic polynomials with constant coefficients and with degrees $n_{a H}$ and $n_{b H}$ respectively:

$$
\begin{aligned}
& A_{H_{0}}\left(q^{-1}\right)=1+a_{H, 1}^{0} q^{-1}+\ldots+a_{H, n_{a H}}^{0} q^{-n_{a H}}, \\
& B_{H_{0}}\left(q^{-1}\right)=1+b_{H, 1}^{0} q^{-1}+\ldots+b_{H, n_{b H}}^{0} q^{-n_{b H}},
\end{aligned}
$$

and with respective degrees $n_{a H}$ and $n_{b H}$. The noise process $H_{0}\left(q^{-1}\right)$ is assumed to be stable and to have a stable inverse. In case $A_{H_{0}}\left(q^{-1}\right)=B_{H_{0}}\left(q^{-1}\right)=1$, (4) defines an OE noise model, whereas with the representation given by (1a-b), (5) is general enough to represent BJ-type of noise models.

For closed-loop identification, Fig. 2, the open-loop data generating system (1) is used with the the noise process given by (5), the reference signals $r_{1}(k)$ and $r_{2}(k)$ as depicted in Fig. 2 and a stabilizing LTI controller $K_{0}$ given by

$$
K_{0}\left(q^{-1}\right)=\frac{B_{K_{0}}\left(q^{-1}\right)}{A_{K_{0}}\left(q^{-1}\right)},
$$

where $B_{K_{0}}\left(q^{-1}\right)$ and $A_{K_{0}}\left(q^{-1}\right)$ are polynomials of respective degrees $n_{b K}$ and $n_{a K}$, $n_{a K} \geq n_{b K}$, respectively given by

$$
\begin{aligned}
& B_{K_{0}}\left(p_{k}, q\right)=b_{K, 0}^{0}+b_{K, 1}^{0} q^{-1}+\ldots+b_{K, n_{b K}}^{0} q^{-n_{b K}}, \\
& A_{K_{0}}\left(p_{k}, q\right)=1+a_{K, 1}^{0} q^{-1}+\ldots+a_{K, n_{a K}}^{0} q^{-n_{a K}} .
\end{aligned}
$$

Journal of Engineering Sciences, Assiut University, Faculty of Engineering, Vol. 41, No. 4, July, 2013,E-mail address: jes@aun.edu.eg 
Mustafa Rabeei, et al., Lpvioid - a LPV identification toolbox for matlab: recent and novel techniques, pp. 1637 - 1659

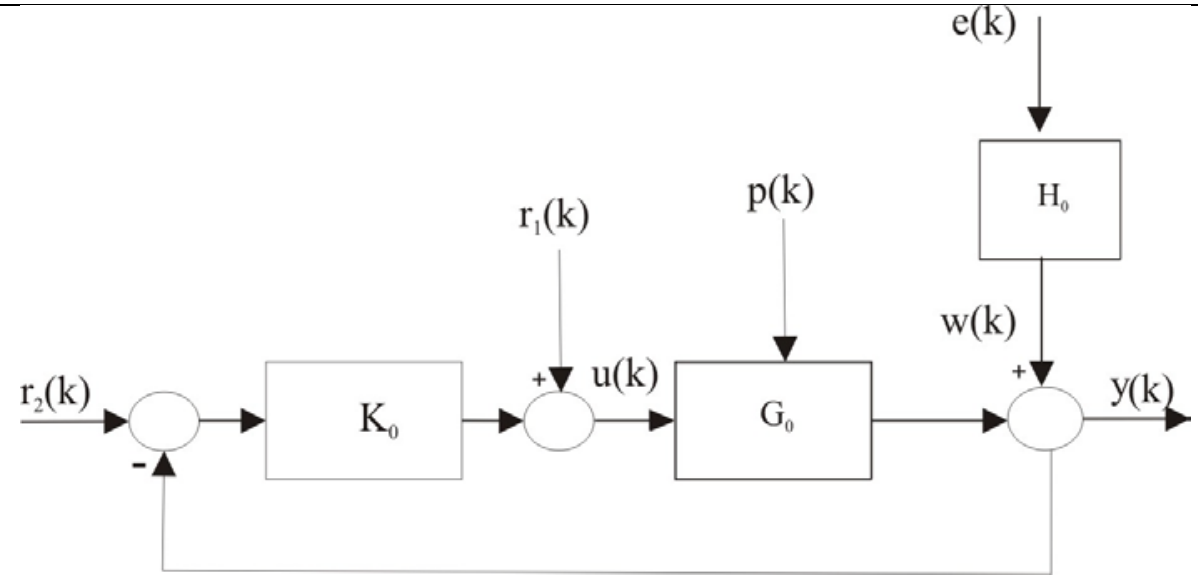

Fig. 2. Closed-loop system

The controller $K_{0}$ guarantees the stability of the closed-loop system for all $p(k) \in \mathrm{P}_{p}$. Stability of such closed-loop system can be assessed using the stability concepts of [6] in Lyapunov sense. Furthermore, $K_{0}$ is assumed to be known.

\section{2. Model representation}

Next the parametrization and structure of the model that identifies the data-generating system (1) with the noise model (4) are introduced, where the process model and the noise model are parametrized separately. The process model is denoted by $G\left(p_{k}, q^{-1}, \theta\right)$ and defined in LPV-IO representation by

$$
A\left(p_{k}, q^{-1}, \theta\right) \hat{y}_{0}(k)=B\left(p_{k}, q^{-1}, \theta\right) q^{-\tau} u(k),
$$

where $A(\cdot)$ and $B(\cdot)$ are polynomials of order $n_{a}$ and $n_{b}$, respectively, given in a form similar to that in (4.1a-b) and with parameter dependent coefficients $a_{i}\left(p_{k}\right)$ and $b_{j}\left(p_{k}\right)$ parametrized respectively by

$$
\begin{array}{r}
a_{i}\left(p_{k}\right)=a_{i, 0}+\sum_{l=1}^{n_{f}} a_{i, l} f_{l}\left(p_{k}\right), \quad i=1, \cdots, n_{a}, \\
b_{j}\left(p_{k}\right)=b_{j, 0}+\sum_{m=1}^{n_{g}} b_{j, m} g_{m}\left(p_{k}\right), \quad j=1, \cdots, n_{b},
\end{array}
$$

Journal of Engineering Sciences, Assiut University, Faculty of Engineering, Vol. 41, No. 4, July, 2013,E-mail address: jes@aun.edu.eg 
Mustafa Rabeei, et al., Lpvioid - a LPV identification toolbox for matlab: recent and novel techniques, pp. 1637 - 1659

with $f_{l}(\cdot), l=1, \cdots, n_{f}$ and $g_{m}(\cdot), m=1, \cdots, n_{g}$ are arbitrary basis functions of $p(k)$ with static dependence and allow the identifiability of the model, see [8]. The process model parameters are collected in the vector

$$
\begin{aligned}
& \theta=\left[a_{1,0} \ldots a_{1, n_{f}} \ldots a_{i, 0} \ldots a_{i, n_{f}} \ldots \ldots a_{n_{a}, n_{f}}\right. \\
& \left.b_{1,0} \ldots b_{1, n_{g}} \ldots b_{j, 0} \ldots b_{j, n_{g}} \ldots \ldots b_{n_{b}, n_{g}}\right]^{\mathrm{T}} \in \mathrm{R}^{n_{\theta}} \text {, } \\
& \text { where } n_{\theta}=n_{a}\left(n_{f}+1\right)+n_{b}\left(n_{g}+1\right) \text {. }
\end{aligned}
$$

The noise-model part of (1), denoted by $H\left(q^{-1}, \rho\right)$, is defined by

$$
H\left(q^{-1}, \rho\right)=\frac{B_{H}\left(q^{-1}, \rho\right)}{A_{H}\left(q^{-1}, \rho\right)} e(k),
$$

where $A_{H}\left(q^{-1}\right)$ and $B_{H}\left(q^{-1}\right)$ are monic polynomials given by a form similar to that in (1a-b) with constant coefficients collected in the vector

$$
\rho=\left[a_{H, 1} \ldots a_{H, n_{a H}} b_{H, 1} \ldots b_{H, n_{b H}}\right]^{\mathrm{T}} \in \mathrm{R}^{n \rho} .
$$

Introduce the model sets,

$$
\mathrm{G}=\left\{G_{\theta} \mid \theta \in \mathrm{R}^{\mathrm{n} \theta}\right\}
$$

consisting of all process models in the form (11),

$$
\mathrm{H}=\left\{H_{\rho} \mid \rho \in \mathrm{R}^{n} \rho\right\}
$$

as the collection of all noise models in the form (15) and

$$
\mathrm{M}=\left\{\left(G_{\theta}, H_{\rho}\right) \mid \operatorname{col}(\theta, \rho)=\Theta \in \mathrm{R}^{n \theta^{+n} \rho}\right\}
$$

\section{3. Identification problem}

Next, the problem of identifying the process and noise models is demonstrated in openand closed-loop settings.

\section{3. 1. Open-loop}

Now (1) with the parametrization (8) and (11) can be written in a linear regression form by

$$
y(k)=\phi^{\mathrm{T}}(k) \theta+\widetilde{v}(k),
$$

where

$$
\widetilde{v}(k)=A\left(p(k), q^{-1}\right) H\left(q^{-1}\right) e(k),
$$

$\phi(k)$ is the regression vector given by

$$
\phi(k)=\left[-y(k-1),,-f_{n_{f}}\left(p_{k}\right) y(k-1),-f_{n_{f}}\left(p_{k}\right) y\left(k-n_{a}\right),\right.
$$

Journal of Engineering Sciences, Assiut University, Faculty of Engineering, Vol. 41, No. 4, July, 2013,E-mail address: jes@aun.edu.eg 
Mustafa Rabeei, et al., Lpvioid - a LPV identification toolbox for matlab: recent and novel techniques, pp. 1637 - 1659

$$
\left.u\left(k-\tau_{d}\right), \cdots g_{n_{g}}\left(p_{k}\right) u\left(k-\tau_{d}\right), g_{n_{g}}\left(p_{k}\right) u\left(k-\tau_{d}-n_{b}\right)\right]^{\mathrm{T}},
$$

and $\theta$ is given by (10). The problem of estimating $\theta$ can be formulated as a minimization of the identification criterion

$$
M\left(\mathrm{D}_{N}, \theta\right)=\frac{1}{N} \sum_{k=1}^{N} \varepsilon_{\theta}^{2}(k),
$$

where $\mathrm{D}_{N}=\{y(k), u(k), p(k)\}, k=1,2, \ldots, N$, denotes a data set collected from the open-loop system (1),

$$
\varepsilon_{\theta}(k)=y(k)-\phi^{\mathrm{T}}(k) \theta
$$

is the prediction error, such that the estimated parameter vector is

$$
\hat{\theta}_{N}=\arg \min _{\theta} M\left(\mathrm{D}_{N}, \theta\right) \text {. }
$$

Estimation of $\theta$ according to (19) is determined under the following assumptions:

A1 The data-generating system belongs to the set of all candidate models, i.e. $\left\{G_{0}, H_{0}\right\} \in \mathrm{M}$, see (15).

A2 The parameterization (9a-b) of the polynomials $A$ and $B,\left\{f_{l}\left(p_{k}\right)\right\}_{l=1}^{n} f$ and $\left\{g_{m}\left(p_{k}\right)\right\}_{m=1}^{n_{g}}$ are chosen such that the model $\left\{G_{\theta}, H_{\rho}\right\}$ is identifiable [8].

A3 The scheduling signal $P$ is noise free.

A4 The data set $D_{N}$ is informative w.r.t the considered model set $M$, [8].

The identification problem can be solved by the least squares approach as follows:

$$
\theta_{l s}=\theta_{0}+\left[\frac{1}{N} \sum_{k=1}^{N} e(k) \phi^{T}(k)\right]\left[\frac{1}{N} \sum_{k=1}^{N} \phi(k) \phi^{T}(k)\right]^{-1} .
$$

Remark 1. For the estimate $\theta_{l s}$ to be consistent, i.e. ${ }^{\theta_{l s}}$ converges in probability to $\theta$, it is necessary that [10]

(i) $\lim _{N \rightarrow \infty} \frac{1}{N} \sum_{k=1}^{N} \phi(k) \phi^{T}(k)$ benonsingular

(ii) $\lim _{N \rightarrow \infty} \frac{1}{N} \sum_{k=1}^{N} \tilde{v}(k) \phi^{T}(k)=0$.

The first condition in (23) is a persistency of excitation condition, which requires that the data set $D_{N}$ be informative [13]. While the second condition can be illustrated as follow: If $\tilde{v}(k)$ is white noise, it will be independent of all past data values, and that condition will be satisfied, whereas in case $\tilde{v}(k)$ is colored noise, it will be correlated with

Journal of Engineering Sciences, Assiut University, Faculty of Engineering, Vol. 41, No. 4, July, 2013,E-mail address: jes@aun.edu.eg 
Mustafa Rabeei, et al., Lpvioid - a LPV identification toolbox for matlab: recent and novel techniques, pp. 1637 - 1659

the delayed output variables present in $\phi(k)$ and second condition will not be satisfied, see [12]. The least squares can give optimal solution for open-loop problem if the noise model is an ARX type, see [10],[12]. In practice noise models are usually not ARX; hence LS is not in general optimal and it will not give consistent estimate, but it is a primary step in all advanced identification methods, like IV and RIV.

\subsubsection{Closed-loop}

For the closed-loop case, see Fig. 2, let $\mathrm{D}_{N}^{c l}=\{y(k), u(k), p(k)\}, k=1,2, \ldots, N$, denote a data set for the closed loop system, with the signals $y(k), p(k)$ are measured and $u(k)$ computed as $u(k)=r_{1}(k)+K_{0}\left(r_{2}(k)-y(k)\right)$. The identification problem can be stated as follows: Given the true closed-loop system shown in Fig. 2, based on the model structure defined in (8) and (11), use the data set $D_{N}^{c l}$ to estimate the model parameters collected in $\theta$ such that assumptions $A 1-A 4$ hold and the controller $K_{0}$ being known and ensure the strict stability of the closed-loop system for all values of the scheduling variable, $p(k)$.

Based on the data set $D_{N}^{c l}$ it is possible to represent the model in a linear regression as in (16-18). Therefore the problem of estimating $\theta$ can be formulated as a minimization of the identification criterion

$$
M\left(\mathrm{D}_{N}^{c l}, \theta\right)=\frac{1}{N} \sum_{k=1}^{N} \varepsilon_{\theta}^{2}(k),
$$

where $\varepsilon_{\theta}(k)$ is the prediction error. Then $\theta$ can be computed by (22), taking into consideration Remark 1.

\section{4. Instrumental variables method}

Due to the inconsistency of the LS estimation when OE and BJ model structures are considered, i.e. bias in the identified parameters; the instrumental variables method is usually used in practice, see [12] [13]. The IV method is able to identify consistently openloop and closed-loop models if the instruments are not correlated with the measurement noise [5]. However, the variance of the estimated parameters will be larger than that with LS estimation. A basic IV method is given by Algorithm 1.

Journal of Engineering Sciences, Assiut University, Faculty of Engineering, Vol. 41, No. 4, July, 2013,E-mail address: jes@aun.edu.eg 
Mustafa Rabeei, et al., Lpvioid - a LPV identification toolbox for matlab: recent and novel techniques, pp. 1637 - 1659

\section{Algorithm 1: One-step IV}

Step 1. Use the LS method to minimize (21) based on the extended regressor (22). This estimates an auxiliary model.

Step 2. Generate an estimate $\hat{y}(k)$ of $y(k)$ using the auxiliary model of Step 1.

Step 3. Construct an instrument based on $\hat{y}(k)$, then estimate $\theta$ using the IV method.

An auxiliary model, for example computed by LS, can be used to generate the instrumental variables vector, as shown in Algorithm 4.4, see [5] for more details.

\subsection{Refined instrumental variables methods, RIV}

The refined instrumental variables method, for open-loop and closed-loop LPV-IO identification has been introduced in [8] and [14], respectively. The method can deliver minimum variance and minimum bias (optimal estimate), however, it needs some iterations. In this method the noise model is identified along with the process model, therefore it assumes a BJ type of noise model, in the form indicated by (4) and (5). By assuming an OE type of noise model the RIV method is simplified to the SRIV method. It is in general suboptimal method, but experiments show that it still delivers small bias and variance, moreover it can be used to identify BJ type model, see [8] and [14] for more details.

\section{Identification of unstable LPV-IO models}

The identification methods discussed in the previous section are used to identify stable LPV-IO models in open- or closed-loop. In this section we propose a technique that is capable to identify unstable LPV-IO models in closed-loop. It is based on formulating the closed-loop system in a form that can represent the LPV closed-loop identification problem in a linear regression form as the LTI case, which permits the use of least squares LS as well as IV approaches systematically.

\section{1. Data generating system}

Consider the closed-loop discrete-time system shown in Fig. 3, with a strictly proper plant $G_{0}\left(p_{k}, q\right)$, i.e. $n_{b}<n_{a}$, defined by (1), (2) and $K_{0}$ is a feedback stabilizing controller. In contrast to the LPV closed-loop identification techniques of [14] and [2], $G_{0}$ and $K_{0}$ might be unstable systems.

Journal of Engineering Sciences, Assiut University, Faculty of Engineering, Vol. 41, No. 4, July, 2013,E-mail address: jes@aun.edu.eg 
Mustafa Rabeei, et al., Lpvioid - a LPV identification toolbox for matlab: recent and novel techniques, pp. 1637 - 1659

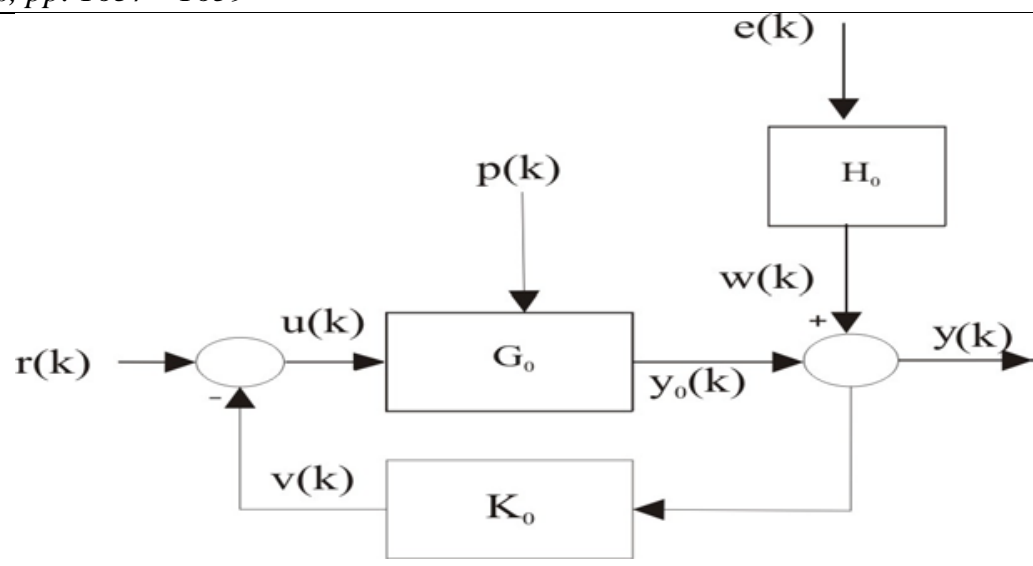

Fig. 3. Closed-loop configuration

We consider the following assumptions:

- The controller $K_{0}$ is a strictly proper LTI system given by

$$
v(k)=K_{0} y(k)=\frac{B_{K_{0}}\left(q^{-1}\right)}{A_{K_{0}}\left(q^{-1}\right)} y(k),
$$

(25)

where $v(k)$ is the controller output and $A_{K_{0}}\left(q^{-1}\right), B_{K_{0}}\left(q^{-1}\right)$ are polynomials of respective degrees $n_{a K}$ and $n_{b K}, n_{a K}>n_{b K}$, given by (7a-b).

- $K_{0}$ guarantees the stability of the closed-loop system for all $p(k) \in \mathrm{P}_{p}$.

- The noise process is represented by

$$
w(k)=H_{0} e(k)=e(k),
$$

where $e(k)$ is a sequence of random variables with zero mean values. This defines an Output Error (OE) noise model.

- The controller output $v(k)$, see Fig. 3, is a noise free signal, i.e. it can be gathered without additive noise.

It is worth to mention that, the plant input $u(k)$ can be considered to be known signal since it is resulted from subtracting two known signals, i.e. $r(k)$ and $v(k)$. Therefore, one can use open-loop identification approach, e.g. [8], to identify the plant from the signal $u(k)$, noise free, and $y(k)$. However, an important condition for open-loop identification is that the identified plant should be stable which is not the case here, as the identified

Journal of Engineering Sciences, Assiut University, Faculty of Engineering, Vol. 41, No. 4, July, 2013,E-mail address: jes@aun.edu.eg 
Mustafa Rabeei, et al., Lpvioid - a LPV identification toolbox for matlab: recent and novel techniques, pp. 1637 - 1659

plant might be unstable. Consequently we carry out the closed-loop identification scheme proposed in this section.

Remark 2. Here we assume both the plant and the controller transfer functions are strictly proper. This assumption is reasonable; since all physical processes are strictly proper by nature.

\section{2. Model representation}

Next the parameterization and structure of the model identifying the data-generating system is introduced. The process model is denoted by $G\left(p_{k}, q^{-1}, \theta\right)$ and defined in inputoutput LPV representation by (8) with $(9(a-b))$. The process model parameters are collected in the parameter vector $\theta$ given by (10). Let $\mathrm{G}=\left\{G_{\theta} \mid \theta \in \mathrm{R}^{n_{\theta}}\right\}$ be the set of all process models in the form (8).

\section{3. Closed-Loop formulation}

Next a representation of the closed-loop system shown in Fig.3 is formulated. Using (1) and (25), the closed-loop system can be written as

$$
\begin{aligned}
{\left[\begin{array}{cc}
A\left(p_{k}, q^{-1}\right) & B\left(p_{k}, q^{-1}\right) \\
-B_{K}\left(q^{-1}\right) & A_{K}\left(q^{-1}\right)
\end{array}\right]\left[\begin{array}{c}
y(k) \\
v(k)
\end{array}\right]=} & \\
{\left[\begin{array}{c}
B\left(p_{k}, q^{-1}\right) \\
0
\end{array}\right] r(k)+\left[\begin{array}{c}
A\left(p_{k}, q^{-1}\right) \\
0
\end{array}\right] w(k), } &
\end{aligned}
$$

where $B_{K}\left(q^{-1}\right)=B_{K_{0}}\left(q^{-1}\right)$ and $A_{K}\left(q^{-1}\right)=A_{K_{0}}\left(q^{-1}\right)$, see (1). Equation (27) can be represented in a compact form by

$A_{c l}\left(p_{k}, q^{-1}\right) x(k)=B_{c l}\left(p_{k}, q^{-1}\right) r(k)+H_{c l}\left(p_{k}, q^{-1}\right) w(k)$,

where

$x(k)=\left[\begin{array}{ll}y(k) & v(k)\end{array}\right]^{\mathrm{T}}$,

$A_{c l}(\cdot), B_{c l}(\cdot)$ and $H_{c l}(\cdot)$ are polynomial matrices given respectively as follows

$A_{c l}\left(p_{k}, q^{-1}\right)=\left[\begin{array}{ll}1 & 0 \\ 0 & 1\end{array}\right]+\sum_{i=1}^{n_{\gamma}}\left[\begin{array}{cc}a_{i}\left(p_{k}\right) & b_{i}\left(p_{k}\right) \\ -b_{K, i} & a_{K, i}\end{array}\right] q^{-i}$,

$B_{c l}\left(p_{k}, q^{-1}\right)=\sum_{j=1}^{n_{b}}\left[\begin{array}{c}b_{j}\left(p_{k}\right) \\ 0\end{array}\right] q^{-j}$,

Journal of Engineering Sciences, Assiut University, Faculty of Engineering, Vol. 41, No. 4, July, 2013,E-mail address: jes@aun.edu.eg 
Mustafa Rabeei, et al., Lpvioid - a LPV identification toolbox for matlab: recent and novel techniques, pp. 1637 - 1659

$$
H_{c l}\left(p_{k}, q^{-1}\right)=\left[\begin{array}{l}
1 \\
0
\end{array}\right]+\sum_{l=1}^{n_{a}}\left[\begin{array}{c}
a_{l}\left(p_{k}\right) \\
0
\end{array}\right] q^{-l},
$$

where $n_{\gamma}=\max \left(n_{a}, n_{a K}\right)$. Note that the controller parameters, $a_{K, 1}, \ldots, a_{K, n_{a K}}$ and $b_{K, 1}, \cdots, b_{K, n_{b K}}$ might be unknown, therefore they can be identified simultaneously with the plant parameters. For generality, we consider that the controller parameters are unknown and the order of the controller is known. For simplicity we assume in (9) that $f_{l}(\cdot)=g_{m}(\cdot), l=1, \cdots, n_{f}$. Now (28) can be written in a linear regression form by

$$
x(k)=\theta^{\mathrm{T}} \phi(k)+\tilde{w}(k),
$$

where

$$
\begin{aligned}
& \tilde{w}(k)=H_{c l}\left(p_{k}, q^{-1}\right) w(k), \\
& \phi(k)=\left[-x^{\mathrm{T}}(k-1),,-f_{n_{f}}(k) x^{\mathrm{T}}\left(k-n_{\gamma}\right), r(k-1),, f_{n_{f}} r\left(k-n_{b}\right)\right]^{\mathrm{T}} \\
& \quad \quad \text { and } \\
& \quad \theta=\left[\alpha_{1,0}, \ldots, \alpha_{1, n_{f}}, \ldots, \alpha_{n_{\gamma}, 0}, \ldots, \alpha_{n_{\gamma}, n_{f}},\right. \\
& \left.\beta_{1,0}, \ldots, \beta_{1, n_{f}}, \ldots, \beta_{n_{b}, 0}, \ldots, \beta_{n_{b}, n_{f}}\right]^{\mathrm{T}},
\end{aligned}
$$

where

$$
\begin{aligned}
& \alpha_{i, l}=\left[\begin{array}{cc}
a_{i, l} & b_{i, l} \\
-b_{K, i} & a_{K, i}
\end{array}\right], i=1,2, \ldots, n_{\gamma}, l=0,1, \ldots, n_{f}, \\
& \beta_{j, l}=\left[\begin{array}{c}
b_{j, l} \\
0
\end{array}\right], j=1,2, \ldots, n_{b}, l=0,1, \ldots, n_{f},
\end{aligned}
$$

\section{4. Identification problem}

Let $\mathrm{D}_{N}^{c l u}=\{x(k), r(k), p(k)\}, k=1,2, \ldots, N$, denote a data set gathered from the data generating system. The identification problem can be stated as follows: Given the true closed-loop system shown in Fig. 3, based on the model structure defined in (28), use the data set $\mathrm{D}_{N}^{\text {clu }}$ to estimate the model parameters collected in $\theta$ (34) under the conditions A1-A4, see section 2.3.1, as well as the following assumptions,

A5 The controller output signal $v(k)$ is noise free.

Journal of Engineering Sciences, Assiut University, Faculty of Engineering, Vol. 41, No. 4, July, 2013,E-mail address: jes@aun.edu.eg 
Mustafa Rabeei, et al., Lpvioid - a LPV identification toolbox for matlab: recent and novel techniques, pp. 1637 - 1659

A6 If the controller is to be identified, its order and structure should be always chosen equal to the true one.

\section{5. Basic IV method}

In prediction error method, [10], with $\tilde{w}$ is a white noise, i.e. $H_{c l}=[10]^{\mathrm{T}}$, the model represented in (28) yields a one-step-ahead predictor

$$
\hat{x}_{\theta}(k \mid k-1)=\theta^{\mathrm{T}} \phi(k),
$$

which provides a one-step-ahead prediction error

$$
\varepsilon_{\theta}(k)=x(k)-\hat{x}_{\theta}(k \mid k-1) .
$$

Therefore the problem of estimating $\theta$ can be formulated as a minimization of the identification criterion

$$
M\left(\mathrm{D}_{N}^{c l u}, \theta\right)=\frac{1}{N} \sum_{k=1}^{N} \varepsilon_{\theta}^{2}(k)
$$

such that the parameter estimation is

$$
\hat{\theta}_{N}=\arg \min _{\theta} M\left(\mathrm{D}_{N}^{c l u}, \theta\right) \text {. }
$$

By considering (31) with the extended regressor in (37), the use of the LS method to obtain the solution (40) leads to optimal estimates of $\theta$. In case that $\tilde{w}$ is not a white noise as the situation here, see (32), IV methods can be used to provide unbiased estimates if the instrument is not correlated to the measurement noise, [5]. However, the variance of the estimated parameters will be larger than that of the LS estimation. Consider the assumptions A1-A6 in the previous subsection, a one-step IV algorithm is given by Algorithm 1. The problem of identifying the model (28) can be seen as an identification of an LPV model in open-loop.

\subsection{Iterative algorithm}

Next an alternative method is presented to identify consistently the model (28). The model (28) can be rewritten as

$$
A_{c l}\left(p_{k}, q^{-1}\right) x(k)=B_{c l}\left(p_{k}, q^{-1}\right) r(k)+\tilde{H}_{c l}\left(p_{k}, q^{-1}\right) \breve{w}(k)
$$

where

$$
\tilde{H}_{c l}\left(p_{k}, q^{-1}\right)=\left[\begin{array}{cc}
A\left(p_{k}, q^{-1}\right) & 0 \\
0 & 1
\end{array}\right], \quad \breve{w}(k)=\left[\begin{array}{c}
w(k) \\
0
\end{array}\right]
$$

Therefore, a one step ahead predictor for the model (41) can be formulated, [13], by

$$
\begin{aligned}
& \hat{x}_{\theta}(k \mid k-1)=B_{c l}\left(p_{k}, q^{-1}\right) r(k)+\left(I_{2}-A_{c l}\left(p_{k}, q^{-1}\right)\right) x(k) \\
& +\left(\tilde{H}_{c l}\left(p_{k}, q^{-1}\right)-I_{2}\right) \varepsilon_{\theta}(k)
\end{aligned}
$$

Journal of Engineering Sciences, Assiut University, Faculty of Engineering, Vol. 41, No. 4, July, 2013,E-mail address: jes@aun.edu.eg 
Mustafa Rabeei, et al., Lpvioid - a LPV identification toolbox for matlab: recent and novel techniques, pp. 1637 - 1659

where $I_{2} \in \mathrm{R}^{2 \times 2}$ is the identity matrix and the prediction error $\varepsilon_{\theta}(k)$ is given by (38). The predictor (42) can be reprinted in a pseudolinear regression by

$$
\begin{aligned}
& \hat{x}_{\theta}(k \mid k-1)=\theta_{E}^{\mathrm{T}} \phi\left(k \mid \theta_{E}\right), \\
& \text { where } \begin{array}{r}
\theta_{E} \text { is extended with the parameters of } \tilde{H}_{c l}(\cdot) \text { as follows } \\
\qquad \theta_{E}=\left[\alpha_{1,0}, \ldots, \alpha_{1, n_{f}}, \ldots, \alpha_{n_{\gamma}, 0}, \ldots, \alpha_{n_{\gamma}, n_{f}},\right.
\end{array} \\
& \left.\beta_{1,0}, \ldots, \beta_{1, n_{f}}, \ldots, \beta_{n_{\delta}, 0}, \ldots, \beta_{n_{\delta}, n_{f}}, \omega_{1,0}, \cdots, \omega_{n_{a}, n_{f}}\right]^{\mathrm{T}}, \\
& \omega_{i, l}=\left[\begin{array}{rr}
a_{i, l} & 0 \\
0 & 1
\end{array}\right], i=1,2, \ldots, n_{a}, l=0,1, \ldots, n_{f}, \\
& \text { and } \phi(k) \begin{array}{r}
\text { is an extension of }(33) \text { given by } \\
\phi(k)=\left[-x^{\mathrm{T}}(k-1),,-f_{n_{f}}(k) x^{\mathrm{T}}\left(k-n_{\gamma}\right), r(k-1),\right. \\
\left., f_{n_{f}} r\left(k-n_{b}\right), \varepsilon_{\theta}^{\mathrm{T}}(k-1), f_{n_{f}} \varepsilon_{\theta}^{\mathrm{T}}\left(k-n_{a}\right)\right]^{\mathrm{T}}
\end{array}
\end{aligned}
$$

An iterative LS algorithm, inspired by the approach of [13], is introduced here to minimize the identification criterion (39) corresponding to (43), see Algorithm 2.

\section{Algorithm 2: Iterative LS identification}

1. Use the LS method to minimize (39) based on the regressor (33) to estimate an ARX auxiliary model. Set $\tau=0$.

2. repeat

3. Generate an estimate $\hat{e}_{\theta}^{(\tau)}(k)$ of $e_{\theta}(k)$ using the resulting model in the previous Step, using (38).

4. Build the extended regressor $\phi_{\tau}(k)$ using (46).

5. Estimate $\theta$ in term of $\hat{\theta}_{(\tau+1)}^{\mathrm{T}}=\left[\frac{1}{N} \sum_{k=1}^{N} x(k) \phi_{\tau}^{\mathrm{T}}(k)\right]\left[\frac{1}{N} \sum_{k=1}^{N} x(k) \phi_{\tau}(k) \phi_{\tau}^{\mathrm{T}}(k)\right]^{-1}$. Increase $\tau$ by 1.

6. until $\theta_{\tau}$ has converged or maximum number of iterations is reached.

7. return estimated plant (and controller) parameters.

Journal of Engineering Sciences, Assiut University, Faculty of Engineering, Vol. 41, No. 4, July, 2013,E-mail address: jes@aun.edu.eg 
Mustafa Rabeei, et al., Lpvioid - a LPV identification toolbox for matlab: recent and novel techniques, pp. 1637 - 1659

\section{LPVIOID overview}

The GUI (Graphical User Interface) of LPVIOID toolbox is opened by typing LPVIOID in the MATLAB command window. This opens the main window shown in Fig. 4.

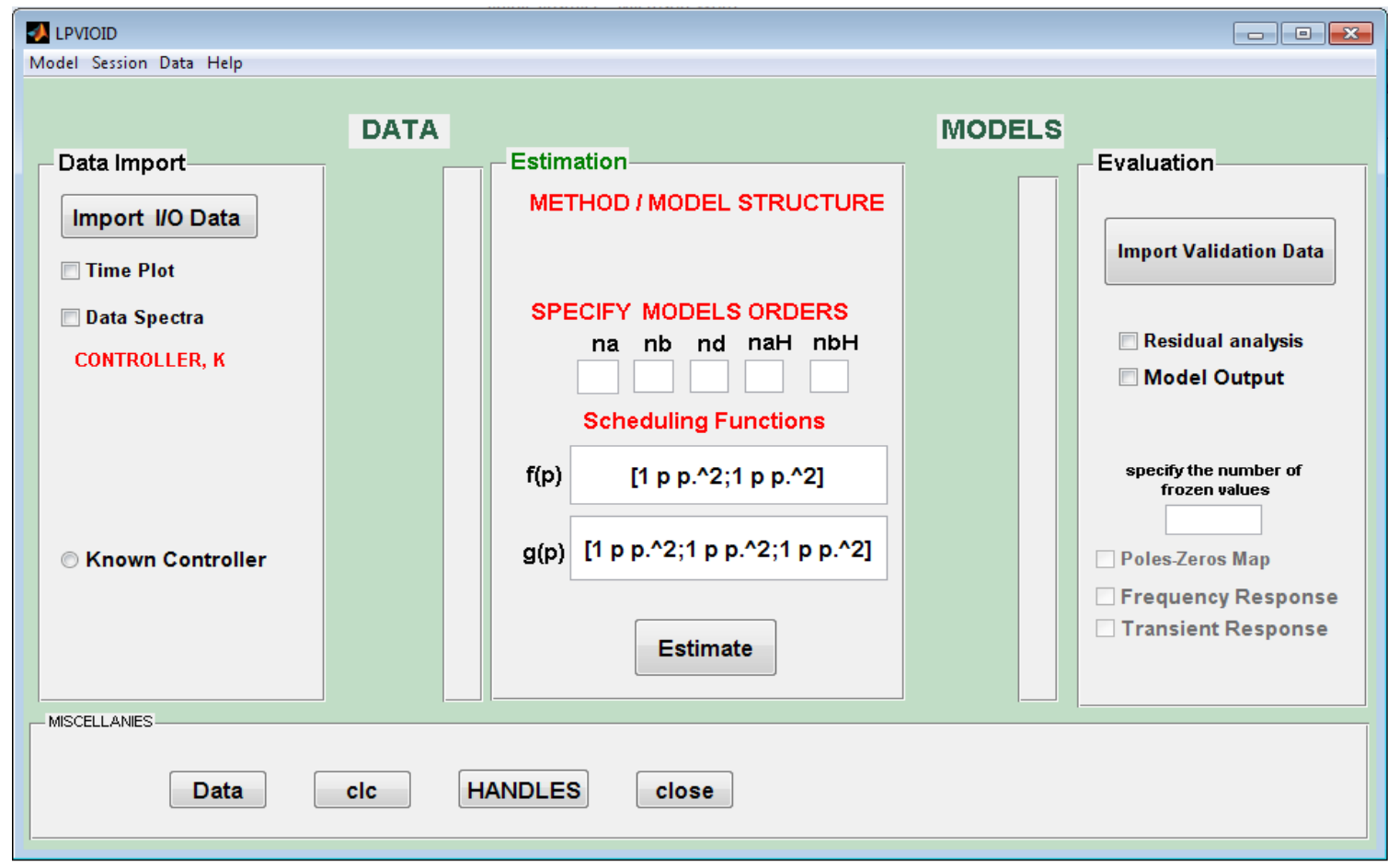

Fig. 4. LPVIOID main window

By looking to the main window we can find how much it is easy to perform the identification of models from a set of measurement data. The user starts by clicking on the Import I/O Data button which raises up a special window that allow specifying the input, output and scheduling signals, from available MATLAB workspace variables. Then the user turns to the estimation area where he/she specifies the identification technique, the orders of the model to be estimated as well as the scheduling functions. Finally the estimated model is represented in the evaluation area where it can be selected by the user to perform different validation and evaluation procedures in time and frequency domains. In the following we shall illustrate the main parts of LPVIOID main window:

1. A data board on the left part, where a data set can be imported from MATLAB workspace or a data file. The imported data set is represented by a colored line icon that can be selected by a mouse action. For closed-loop identification, the controller numerator and denominator coefficients can be typed or imported.

Journal of Engineering Sciences, Assiut University, Faculty of Engineering, Vol. 41, No. 4, July, 2013,E-mail address: jes@aun.edu.eg 
Mustafa Rabeei, et al., Lpvioid - a LPV identification toolbox for matlab: recent and novel techniques, pp. 1637 - 1659

2. An estimation board in the middle: This enables the user to select a suitable method among the others and to choose a suitable noise model structure from a list of models. The user can specify the suitable orders, delays and scheduling functions of the identified model.

3. A model evaluation board in the right: This contains tools enable the user to perform different evaluation procedures in time and frequency domains as well as performing residual analysis. For example, by the Model Output option, the quality of the model that has been identified can be assessed. To this end, the predicted output by the identified model is compared with the measured output signal. As a figure of merit, we use the Best Fit Rate (BFR) criterion [9]:

$$
B F R=\max \left(\left(1-\frac{|y(k)-\hat{y}(k, \theta)|_{2}}{|y(k)-\bar{y}|_{2}}\right), 0\right) 100 \%
$$

Where $|\cdot|_{2}$ is the $\ell_{2}$ norm, $\bar{y}$ is the mean of $y(k)$ and $\hat{y}(k, \theta)$ is the simulated model output based on the validation data.

4. The menu bar in the upper part: It contains dedicated sub-menus for each of the following:

- data: deletion, time-domain plot or spectrum analysis.

- model: deletion, evaluation or exporting to MATLAB workspace.

- session: bookkeeping; open, save, save-as or close.

- help: open product help, quick start documents or running quick start Demo video.

For more information about the facilities of the LPVIOID GUI, see the user manual available with the toolbox at the website:

https://sites.google.com/site/mustafarabeei/home/lpvioid-toolbox

\section{Simulation examples}

In this section we present three examples for the identification in open-loop and closedloop using the LPVIOID toolbox.

\section{1. Data generating system}

Consider the data generating system, Fig. 1, that is described by (1) with (2a-b) and with coefficients:

Journal of Engineering Sciences, Assiut University, Faculty of Engineering, Vol. 41, No. 4, July, 2013,E-mail address: jes@aun.edu.eg 
Mustafa Rabeei, et al., Lpvioid - a LPV identification toolbox for matlab: recent and novel techniques, pp. 1637 - 1659

$$
\begin{aligned}
& a_{1}^{o}\left(p_{k}\right)=f_{k, 0}-0.5 f_{k, 1}-0.1 f_{k, 2}, \\
& a_{2}^{o}\left(p_{k}\right)=0.5 f_{k, 0}-0.7 f_{k, 1}-0.1 f_{k, 2}, \\
& b_{1}^{o}\left(p_{k}\right)=0.5 f_{k, 0}-0.4 f_{k, 1}+0.01 f_{k, 2}, \\
& b_{2}^{o}\left(p_{k}\right)=0.2 f_{k, 0}-0.3 f_{k, 1}-0.02 f_{k, 2},
\end{aligned}
$$

where

$$
f_{k, 0}=1, \quad f_{k, 1}=p(k), \quad f_{k, 2}=p^{2}(k) .
$$

The scheduling variable $p(k)$ is given by

$$
p(k)=\eta(0.5 \sin (0.35 \pi k)+0.5) \in[0 \eta]
$$

where $\eta$ is a scalar which takes different values to affect the stability of the plant as shown below. In this part, the robustness of the LPVIOID identification methods are investigated with respect to certain signal-to-noise ratio (SNR)

$$
\operatorname{SNR}=10 \log \left(\frac{P_{y_{0}}}{P_{e}}\right),
$$

where $P_{y_{0}}$ and $P_{e}$ are the average power of signals $y_{0}$ and $e$ respectively, see Fig. 1 .

\section{2. Open-loop identification}

To illustrate open-loop identification the open-loop system (1) with (48-49) is considered with $u(k)$ taken as a white noise with a uniform distribution $U(-1,1), \eta=1$ to make the open-loop system strictly stable and a number of data samples $N=4000$. Furthermore the white noise disturbance $e(k) \in \mathrm{N}\left(0, \mu^{2}\right)$ is considered with $\mu=0.002$, where $\mu^{2}$ is the variance, to produce a $S N R=15 \mathrm{~dB}$ and the following noise model

$$
H(q)=\frac{1+0.5 q^{-1}}{1+0.3 q^{-1}}
$$

is considered. Next the generated data set is imported to the LPVIOID, see section 4, and a model is estimated using the RIV identification method, with $n_{a}=2, n_{b}=1, n_{d}=1, n_{a H}=1$ and $n_{b H}=1$. Finally, the estimated model is evaluated using the Model Output option in the evaluation board and the BFR (47) is found to be $82.388 \%$, see Fig. 5. The RIV method gives a good estimates of both plant and noise model parameters, relative to a $S N R$ of $15 \mathrm{~dB}$, as indicated in table 1 . Other options can be applied to perform more evaluation of the estimated model, see section 4 .

Journal of Engineering Sciences, Assiut University, Faculty of Engineering, Vol. 41, No. 4, July, 2013,E-mail address: jes@aun.edu.eg 
Mustafa Rabeei, et al., Lpvioid - a LPV identification toolbox for matlab: recent and novel techniques, pp. 1637 - 1659

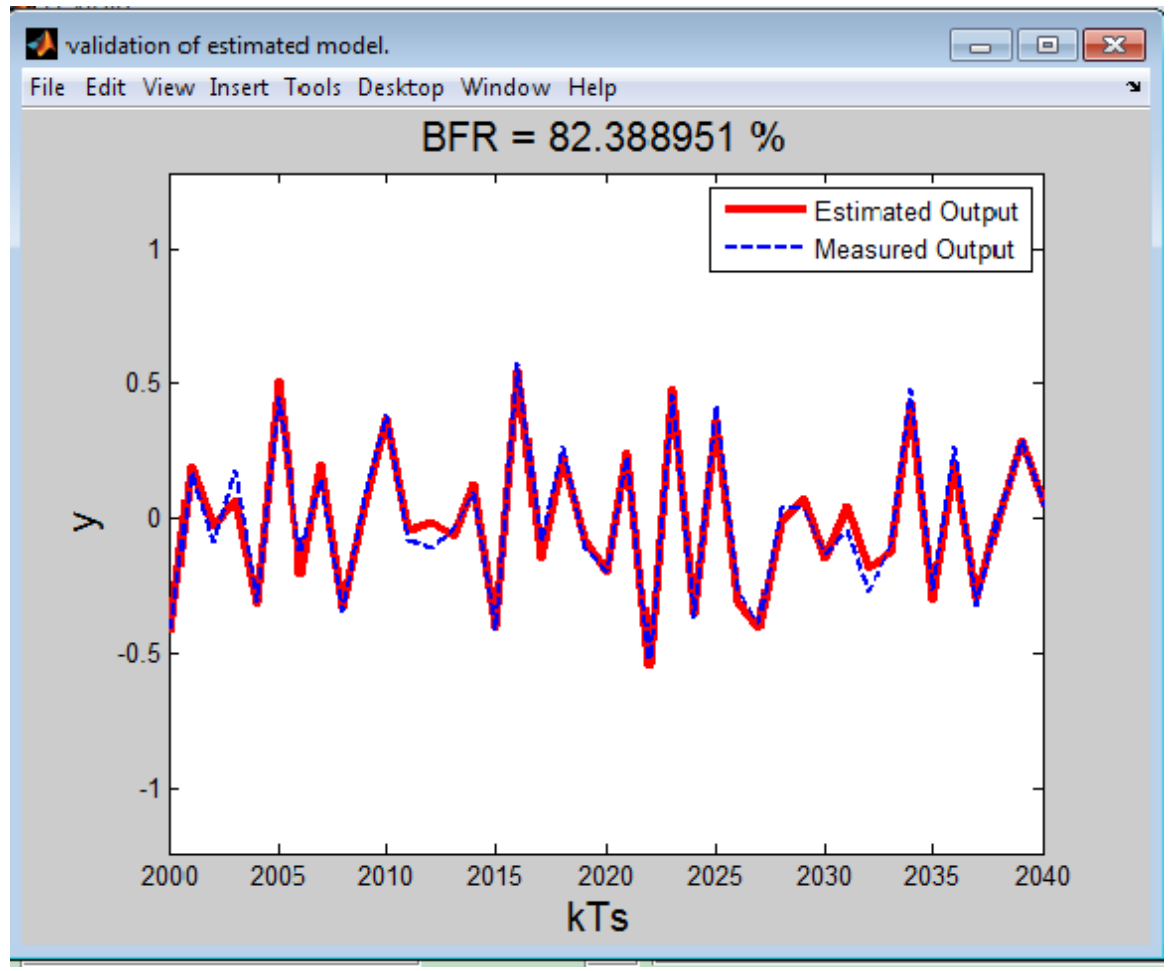

Fig. 5. LPVIOID open-loop model output

Table 1.

Open-loop identification

\begin{tabular}{|c|c|c|c|c|c|c|}
\hline \multicolumn{7}{|c|}{ estimated parameters of the $A$ polynomial } \\
\hline method & $a_{1,1}$ & $a_{1,2}$ & $a_{1,3}$ & $a_{2,1}$ & $a_{2,2}$ & $a_{2,3}$ \\
\hline EXACT & 1 & -0.5 & -0.1 & 0.5 & -0.7 & -0.1 \\
\hline RIV & 0.9939 & -0.5070 & -0.0295 & 0.5088 & -0.7850 & 0.0282 \\
\hline \multicolumn{7}{|c|}{ estimated parameters of the $B$ polynomial } \\
\hline method & $b_{1,1}$ & $b_{1,2}$ & $b_{1,3}$ & $b_{2,1}$ & $b_{2,2}$ & $b_{2,3}$ \\
\hline EXACT & 0.5 & -0.4 & 0.01 & 0.2 & -0.3 & -0.02 \\
\hline RIV & 0.5007 & -0.4060 & 0.0167 & 0.1976 & -0.3107 & $\mathbf{0 . 0 0 8 7}$ \\
\hline \multicolumn{7}{|c|}{ the estimated $A_{H}$ and $B_{H}$ polynomials' parameters } \\
\hline \multicolumn{2}{|c|}{ method } & \multicolumn{2}{|r|}{$a_{H, 1}$} & \multicolumn{3}{|c|}{$b_{H, 1}$} \\
\hline \multicolumn{2}{|c|}{ EXACT } & \multicolumn{2}{|r|}{0.3} & \multicolumn{3}{|c|}{0.5} \\
\hline \multicolumn{2}{|r|}{ RIV } & \multicolumn{2}{|c|}{0.3194} & \multicolumn{3}{|c|}{0.4926} \\
\hline
\end{tabular}

Journal of Engineering Sciences, Assiut University, Faculty of Engineering, Vol. 41, No. 4, July, 2013, E-mail address: jes@aun.edu.eg 
Mustafa Rabeei, et al., Lpvioid - a LPV identification toolbox for matlab: recent and novel techniques, pp. 1637 - 1659

\section{3. Closed-loop identification for stable plants}

For closed-loop identification, see section 2.3, the plant (1) with the coefficients (48-49) is simulated with a stabilizing controller

$$
K(q)=\frac{1+0.5 q^{-1}}{1-0.85 q^{-1}}
$$

and the scheduling variable as given in (50) with $\eta=1$, such that the open-loop and closed-loop systems be stable in the whole scheduling range. In the simulation, the reference signals $r_{1}(k)$ and $r_{2}(k)$ are both taken as white noise with a uniform distribution $\mathrm{U}(-1,1)$ and a number of data sample $N=4000, e(k)$ is taken as a white noise disturbance with uniform distribution $\mathrm{N}\left(0, \mu^{2}\right)$ with $\mu=0.0075$ to produce a SNR $=15 \mathrm{~dB}$ and the noise model (52) is considered. The RIV identification method in LPVIOID is applied, with $n_{a}=2, n_{b}=1, n_{d}=1, n_{a H}=1$ and $n_{b H}=1$. Estimated model parameters are listed in table 1 and the simulated model output is compared in Fig. 6 with the true system output.

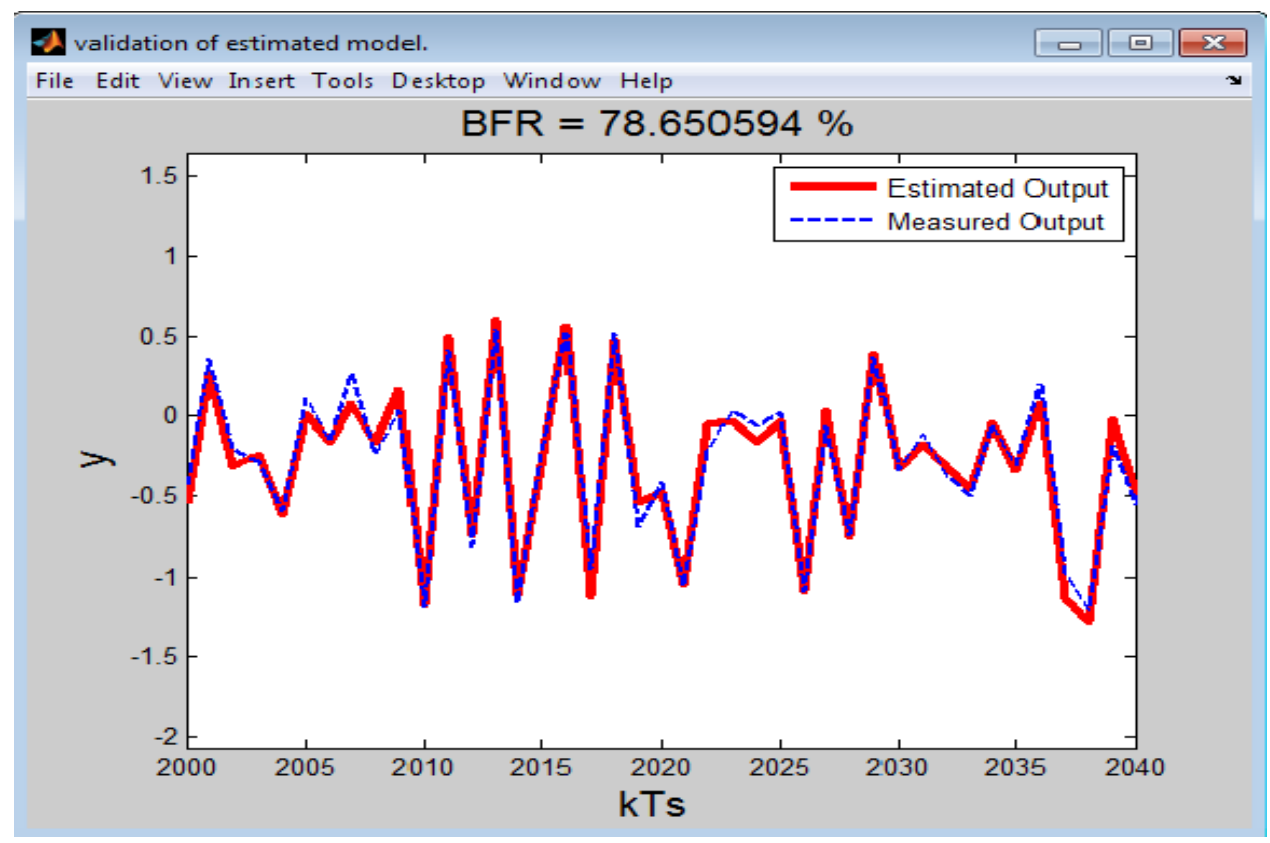

Fig. 6. LPVIOID closed-loop model output

As shown in table 2 and Fig. 6, the RIV method gives good estimation of the plant and the noise-model parameters with a $B F R=78.65$.

Journal of Engineering Sciences, Assiut University, Faculty of Engineering, Vol. 41, No. 4, July, 2013,E-mail address: jes@aun.edu.eg 
1656

Mustafa Rabeei, et al., Lpvioid - a LPV identification toolbox for matlab: recent and novel techniques, pp. 1637 - 1659

\section{Table 2.}

Closed-loop identification

\begin{tabular}{|c|c|c|c|c|c|c|}
\hline \multicolumn{7}{|c|}{ the estimated A polynomial parameters } \\
\hline Method & $a_{1,1}$ & $a_{1,2}$ & $a_{1,3}$ & $a_{2,1}$ & $a_{2,2}$ & $a_{2,3}$ \\
\hline EXACT & 1 & -0.5 & -0.1 & 0.5 & -0.7 & -0.1 \\
\hline RIV & 0.9992 & -0.4441 & -0.1887 & 0.4971 & -0.6503 & -0.1760 \\
\hline \multicolumn{7}{|c|}{ the estimated B polynomial's parameters } \\
\hline Method & $b_{1,1}$ & $b_{1,2}$ & $b_{1,3}$ & $b_{2,1}$ & $b_{2,2}$ & $b_{2,3}$ \\
\hline EXACT & 0.5 & -0.4 & 0.01 & 0.2 & -0.3 & -0.02 \\
\hline RIV & 0.4975 & -0.3943 & 0.0068 & 0.1997 & -0.2891 & -0.0352 \\
\hline \multicolumn{7}{|c|}{ the estimated $A_{H}$ and $B_{H}$ polynomials' parameters } \\
\hline Method & & $a_{H, 1}$ & & $b_{H, 1}$ & & \\
\hline EXACT & & 0.3 & & 0.5 & & \\
\hline RIV & & 0.3767 & & 0.5651 & & \\
\hline
\end{tabular}

\section{4. Closed-loop identification with unstable plant}

Finally, the same plant used above is made unstable, by putting $\eta=1.7$ in the scheduling variable expression (50). To stabilize the closed loop system, see Fig. 3 , for that new range of scheduling variable we use the following controller

$$
K(q)=\frac{0.1 q^{-1}-0.5 q^{-1}}{1+0.1 q^{-1}+0.2 q^{-1}}
$$

Next the closed-loop system is simulated using $r(k)$ taken as a white noise with a uniform distribution $\mathrm{U}(-1,1)$, a number of data samples $N=4000$ and an additive white noise disturbance $e(k) \in \mathrm{N}\left(0, \mu^{2}\right)$ with $\mu=0.0025$, to produce a $S N R=15 d B$. The noise model is taken as $H=1$, i.e. OE type. Estimated parameters using the LS, IV, and iterative-LS methods, explained in section 3, respectively, are shown in table 3 . Using the option Compare under the menu Model, the three estimated models' outputs were plotted in a single figure as shown in Fig. 7 and the BFR of each model is also calculated. From table 3 and Fig. 7 it is found that LS provides biased estimate, iterative LS gives a little better estimate, while, IV gives the best estimate, $B F R=81.226 \%$.

Journal of Engineering Sciences, Assiut University, Faculty of Engineering, Vol. 41, No. 4, July, 2013,E-mail address: jes@aun.edu.eg 
Mustafa Rabeei, et al., Lpvioid - a LPV identification toolbox for matlab: recent and novel techniques, pp. 1637 - 1659

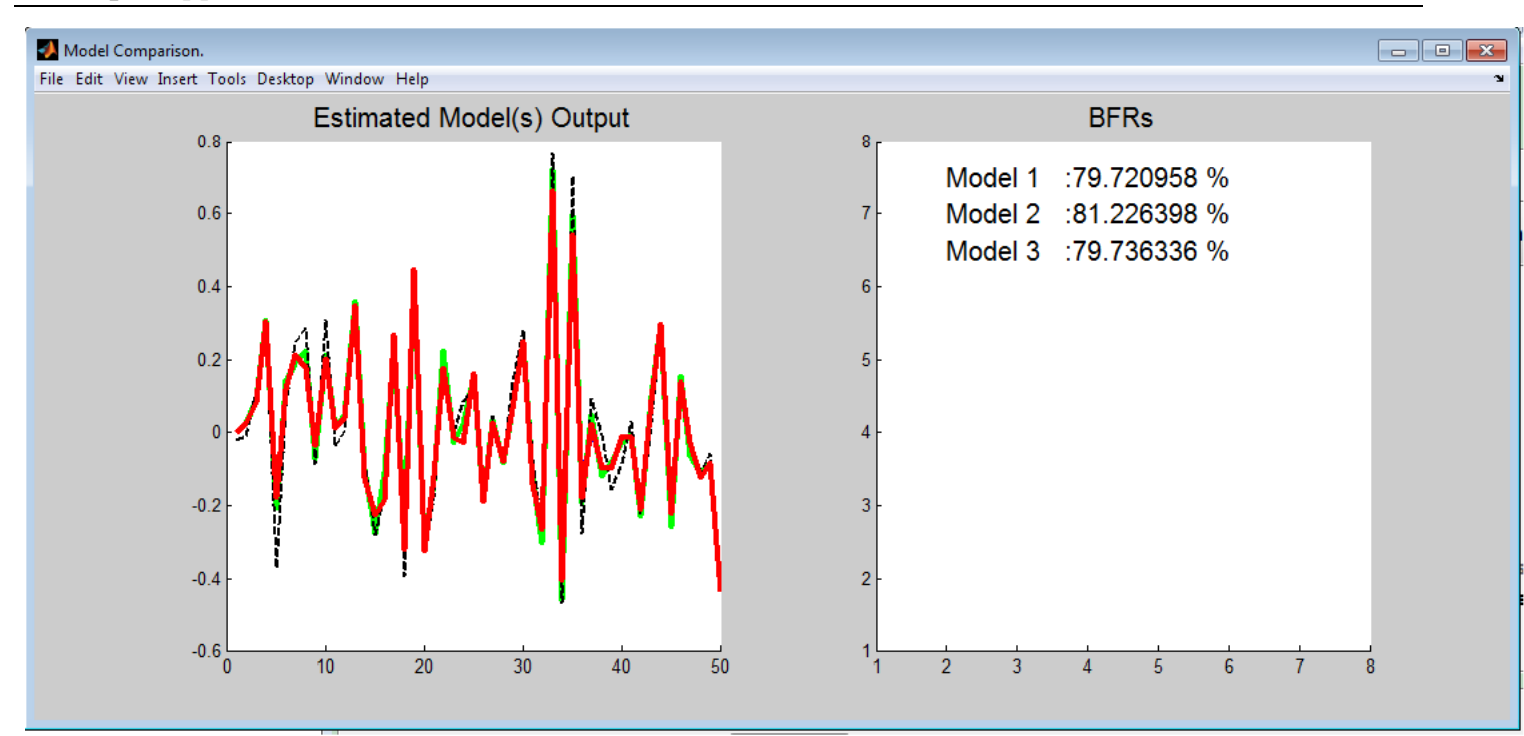

Fig. 7. LPVIOID closed-loop model output

Table 3.

Unstable plant identification

\begin{tabular}{|c|r|r|r|r|r|r|}
\hline \multicolumn{7}{|c|}{ the estimated A polynomial parameters } \\
\hline method & $a_{1,1}$ & $a_{1,2}$ & $a_{1,3}$ & $a_{2,1}$ & $a_{2,2}$ & $a_{2,3}$ \\
\hline EXACT & 1 & -0.5 & -0.1 & .5 & -0.7 & -0.1 \\
\hline LS & 0.7919 & -0.2102 & -0.1691 & 0.3710 & -0.5207 & -0.1183 \\
\hline IV & 0.9825 & -0.4715 & -0.1435 & 0.4799 & -0.7226 & -0.0827 \\
\hline iterative-LS & 0.7923 & -0.2127 & -0.1673 & 0.3706 & -0.5230 & -0.1164 \\
\hline \multicolumn{7}{|c|}{ the estimated B polynomial parameters } \\
\hline method & $b_{1,1}$ & $b_{1,2}$ & $b_{1,3}$ & $b_{2,1}$ & $b_{2,2}$ & $b_{2,3}$ \\
\hline EXACT & .5 & -0.4 & 0.01 & 0.2 & -0.3 & -.02 \\
\hline LS & 0.4959 & -0.3884 & 0.0041 & 0.1390 & -0.2051 & -0.0531 \\
\hline IV & 0.4959 & -0.3863 & 0.0035 & 0.1990 & -0.3138 & -0.0122 \\
\hline iterative-LS & 0.4958 & -0.3890 & 0.0044 & 0.1390 & -0.2045 & -0.0536 \\
\hline
\end{tabular}

Journal of Engineering Sciences, Assiut University, Faculty of Engineering, Vol. 41, No. 4, July, 2013,E-mail address: jes@aun.edu.eg 
Mustafa Rabeei, et al., Lpvioid - a LPV identification toolbox for matlab: recent and novel techniques, pp. 1637 - 1659

\section{Conclusions and future work}

This paper has presented the LPVIOID, a system identification toolbox for MATLAB to identify LPV-IO models. The toolbox comprises the more recent identification methodologies and supported with a user friendly graphical user interface (GUI). Furthermore the toolbox supports both open-/closed-loop identification and includes many options for model evaluation in time and frequency domains. A novel technique for the identification of unstable LPV-IO plants in closed-loop has been introduced and embedded in the LPVIOID. The current version of LPVIOID deals with the SISO LPV models only, an extension to the MIMO case is a straightforward future work.

\section{References}

[1] H. Abbas, M. Ali, and H. Werner. Linear Recurrent Neural Network for open- and closedloop consistent identification of LPV models. In IEEE Conference on Decision and Control (CDC), pages 6851-6856, Atlanta, Georgia, USA, 2010.

[2] A. Ali, M. Ali, and H. Werner. Indirect closed-loop identification of input-output LPV models: A pre-filtering approach. In Proc. of the 18th IFAC World Congress, pages 41864191, Milan, Italy, 2011.

[3] P. Apkarian and P. Gahinet A Convex Characterization of Gain-Scheduled $\mathrm{H}_{\infty}$ Controllers IEEE Trans. Automatic Control, vol. 40, no. 5, pp. 853-864, 1995

[4] B. Bamieh and L. Giarré. Identification of linear parameter varying models. International Journal of Robust and Nonlinear Control, 12(9):841-853, 2002.

[5] M. Butcher, A. Karimi, and R. Longchamp. On the consistency of certain identification methods for linear parameter varying systems. In Proc. of the 17th IFAC World Congress, pages 4018-4023, Seoul, Korea, 2008.

[6] W. Gilbert, D. Henrion, J. Bernussou, and D. Boyer. Polynomial LPV synthesis applied to turbofan engine. IFAC Control Engineering Practice, 18(9):1077-1083, 2010. , Engineering Department, 2002.

[7] A. Kominek, H. Werner, M. Garwon, and M. Schultalbers. Control of Linear Parameter Varying Systems with Applications, chapter Identification of Low-Complexity LPV InputOutput Models for Control of a Turbocharged Combustion Engine. Springer US, 2012.

[8] V. Laurain, M. Gilson, R. Tóth, and H. Garnier. Refined instrumental variable methods for identification of LPV box-jenkins models. Automatica, 46(6):959-967, 2010.

[9] L. Ljung, System Identification Toolbox, for use with Matlab. The Mathworks Inc., 2006.

[10] L. Ljung. System Identification, Theory for the User. Prentice-Hall Inc. USA, 2nd edition edition, 1999.

[11] C.W. Scherer. Robust generalized H2 control for uncertain and LPV systems with general scalings. In Proc. of the 47th IEEE Conference on Decision and Control, Kobe, Japan, 4(0):3970-3975, 1996.

[12] T. Söderström and P. Stoica. Instrumental Variable Methods for System Identification. Springer-Verlag, 1983.

[13] R. Tóth, P. Heuberger, and P. Van den Hof. Control of Linear Parameter Varying Systems with Applications, chapter Prediction-Error Identification of LPV Systems: Present and Beyond. Springer US, 2012.

Journal of Engineering Sciences, Assiut University, Faculty of Engineering, Vol. 41, No. 4, July, 2013,E-mail address: jes@aun.edu.eg 
Mustafa Rabeei, et al., Lpvioid - a LPV identification toolbox for matlab: recent and novel techniques, pp. 1637 - 1659

[14] R. Tóth, V. Laurain, M. Gilson, and H. Garnier. On the closed loop identification of LPV models using instrumental variables. In Proc. of the 18th IFAC World Congress, pages 7773-7778, Milan, Italy, 2011.

[15] V. Verdult. A Kernel Method for Subspace Identification of Multivariable Bilinear Systems. In Ph.D thesis, Univesity of Twente, 2002.

[16] Herbert Werner: Robust control design with Matlab: D.-W. Gu, P.Hr. Petkov, M.M. Konstantinov; Springer-Verlag London Limited, ISBN: 1-85233-983-7. Automatica 42(9): 1619-1620 (2006).

\section{برمجيه تفاعليه للنمذجة باستخدام الماتلاب تضم طرق حديثه \\ و مبتكره في النمذجة المعملية للأنظمة الخطية ذات المعاملات المتغيرة}

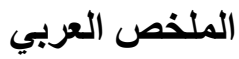

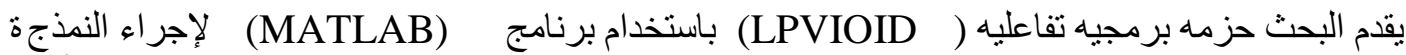

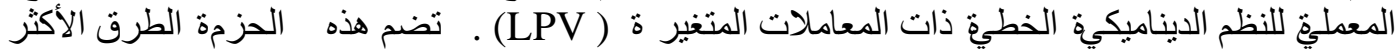

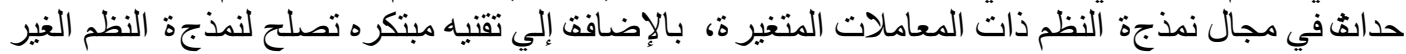

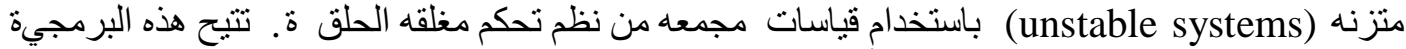

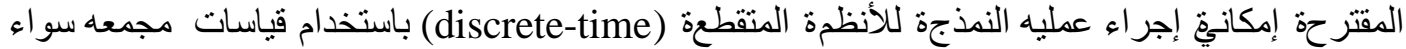

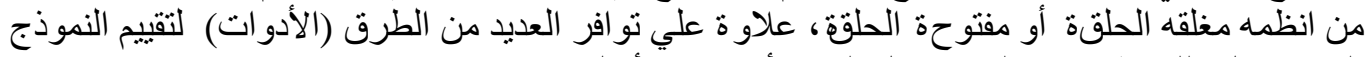
الناتج و كل ذللك بطريقه عمليه. يشمل البحث أيضا عده أمثلة توضيحيه.

Journal of Engineering Sciences, Assiut University, Faculty of Engineering, Vol. 41, No. 4, July, 2013,E-mail address: jes@aun.edu.eg 